\title{
The significant role of quorum sensing in the control of Vibrio cholerae virulence
}

\author{
Bashir Mohammed Abubakar 1, ", Jibrin Ndejiko Mohammed ${ }^{2}$, Abdulrahman Idris ${ }^{3}$, \\ Hamzat Ibiyeye Tijani ${ }^{4}$, Saidu Haruna ${ }^{5}$, Yusuf Hindatu ${ }^{6}$, Mohammed Sulaiman \\ ${ }^{1}$ Department of Biological Sciences, Bauchi State University Gadau, P.M.B 065, Bauchi, Nigeria \\ ${ }^{2}$ Department of Microbiology, Ibrahim Badamasi Babangida University Lapai, P.M.B 011, Niger, Nigeria \\ ${ }^{3}$ Department of Microbiology, Kaduna State University, P.M.B. 2339, Kaduna, Nigeria \\ ${ }^{4}$ Faculty of Bioscience \& Medical Engineering, Universiti Teknologi Malaysia, 81310 Skudai, Johor-bahru, Malaysia \\ ${ }^{5}$ Department of Biological Sciences, Gombe State University, PMB 0127, Gombe, Nigeria \\ ${ }^{6}$ Department of Biochemistry, Bauchi State University Gadau, P.M.B 065, Bauchi, Nigeria
}

\section{Email address:}

elbash1150@yahoo.com (B. M. Abubakar),ndejiko@gmail.com (J. N. Mohammed), saiduharunn@yahoo.com (S. Haruna), janyzrux@gmail.com (H. I. Tijani), yusufhindat@yahoo.com (Y. Hindatu), sumulsu@yahoo.com (M. Sulaiman),

abdurrahman.idris@kasu.edu.ng (A. Idris)

\section{To cite this article:}

Bashir Mohammed Abubakar, Jibrin Ndejiko Mohammed, Abdulrahman Idris, Hamzat Ibiyeye Tijani, Saidu Haruna, Yusuf Hindatu, Mohammed Sulaiman. The Significant Role of Quorum Sensing in the Control of Vibrio Cholerae Virulence. Advances in Bioscience and Bioengineering. Vol. 2, No. 1, 2014, pp. 1-7. doi: 10.11648/j.abb.20140201.11

\begin{abstract}
Quorum sensing (QS) plays a vital role in controlling virulence in bacterial species. Vibrio cholerae, the main causative agent of cholera uses chemical signals to control virulence and biofilm formation. QS Always depend on the secretion and detection of signaling molecules known as auto inducers. Contrary to other bacteria that cause virulence at High cell density (HCD) when they attain a specific threshold, reverse is the case for $V$. Cholerae. At Low cell density (LCD), activation in the expression of virulence gene by $V$. cholerae destabilizes HapR while stabilizing AphA. The activation of AphA structural genes result in the expression of CT and TCP virulence together with the formation of biofilm. At HCD, the coherence of the two quorum-sensing auto-inducers (CAI-1 and AI-2) represses the expression of the virulence genes by activating HapR which in turn synthesizes Hap protease that acts as a detachase to get rid of the virulence genes and biofilm formation. In this review, we outline the QS mechanism used by Vibrio cholerae to cause infection by the production of virulence factors. In addition, we examine how this infection can be minimize through the use of chemicals which act as either inhibitors or agonist to the QS system.
\end{abstract}

Keywords: Virulence, Autoinducers, CAI-1, AI-2, LuxO, HapR, Aph A, QS Inhibitors

\section{Introduction}

Bacteria are capable of responding to a wide range of chemical and physical changes associated with their environment by regulating their gene expression via a phenomenon known as Quorum Sensing (QS). QS plays a vital role in controlling many processes such as biofilm formation, sporulation, bioluminescence, virulence etc. A connection between virulence and QS have already been established in some bacterial species [1] such as in Staphylococcus aureus and Pseudomonas aeruginosa which causes skin infection and cystic fibrosis respectively [2].

QS is a cell-to-cell signaling mechanism in bacteria that involve the production, release and detection of chemical molecules called auto-inducers (AIs) [3]. These AIs are usually released in response to variations in the environmental conditions such as stress, nutrient limitation, shock or high osmolarity which may cause the cell to have mutagenic responses to transcription and translation.

Basically, there are different types of AIs produced by the same species of the same genera or of different genera. Bacteria detect and respond to these chemical signals when they attain a specific threshold by altering their gene expression. This is possible because AIs are secreted at the basal level and their concentration increases with growth. As the signal diffuses through the membrane, the concentration 
inside the cells also increases. When the concentration reaches a specific threshold, the signaling molecules binds to the receptors found within the cells, thereby activating it. These receptors usually influence the expression of the structural genes based on the intensity of the signaling variations. This phenomenon known as QS is always cell density dependent [4]. Bacteria uses QS to regulate different phenotypic activities such as exopolysaccharide production, biofilm formation and virulence functionality $[5,6]$.

\section{Quorum Sensing Discovery}

QS was first discovered in a gram negative bacterium called Vibrio fisheri, a bioluminescent bacterium which co habits in an association with other marine animals [7]. This type of association is known as symbiotic associations which result in the production of light [8]. The light produced by the Vibrio fisheri is used by the host for the purpose of avoiding predators, attracting prey or finding mates [8]. The bacteria produced a diffusible acyl homoserine lactone (AHL) molecule acting as an AIs which is density dependent for specific set of gene expression [9].

The expression of these genes is done by the production of a luciferase enzyme complex which function is to produce light. The luciferase enzyme encoded by $\operatorname{lu} x C D A B E G H$ genes is responsible for the subsequent generation of light which is only expressed at high cell density (HCD) of Vibrio fisheri and repressed at low cell density (LCD) [7]. Thus, the lux CDABEGH structural genes constitute what is referred to as the Lux operon and its transcription and the subsequent translation of its mRNA transcript is only attainable at high bacterial cell density.

\subsection{Types of Auto-Inducers (AIs)}

Basically, there are three major QS circuits or AIs employed by bacteria; these are known as system I and system II. Gram negative and Positive bacteria employed the use of system I, each play a vital role in intra specific communication and finally the third type of circuit is referred to as system II that function in interspecific communication [7]. QS functionality that are specifically employed by both gram positive and gram negative bacteria are known to be intra specific due to their ability to initiate intra species communicating network within the consortia.

\subsection{Quorum Sensing in Gram-Negative LuxIR Circuit}

Acyl homoserine lactones (AHL) is generally used by gram negative LuxIR system for their intra specific communication [10].The biosynthesis of acyl homoserine lactones (AHL) is dependents primarily on the members of the LuxI family of AHL synthases [11] and more than 100 of them are presently found in the bacterial genome database. It was reported by Rutherford and Bassler (2012) that the variety types of AHL produced by different bacterial species have different acyl side chain length ranging from $\mathrm{C} 4$ to $\mathrm{C} 18$.

A protein component called luxI plays a role in the synthesis of oligopeptides signaling molecule (which is AHL in the case of the gram negative bacteria) and has the ability to diffuse across the cell membrane into the extracellular environment. As the concentration of the AIs reaches a specific minimum, they diffuses into the cytoplasm and binds the receptor protein called LuxR to form the LuxIR complex, an activating complex with specific affinity to bind the promoter of the DNA. This concurrent binding activates the transcription of the luxCDABEGH genes [12]. This shows that quorum sensing is cell density dependent. When the microbial cell density is low, the AIs are basically expressed below the minimum threshold. Thus, the Lux operon encoding the luciferase enzyme will not be expressed.

These usually play vital roles in the catalysis of amide bond between charged protein acyl-acyl carrier [11]. The response activity of AHL in the gram negative system takes place within the cytoplasm and exhibits a high level of specificity since AIs synthesized produced by one species do not usually interact with LuxIR complex of other species variants. The auto-inducing molecules fit based on the lock and key model with LuxR protein and there exists no crosstalk within this system because the signaling response activity between the bacteria consortium is only intra specific [4].

\subsection{Quorum Sensing in Gram-Positive Oligopeptide Circuit}

In the gram positive bacteria, the mechanism of QS system have higher resemblance with that of gram negative bacteria but they usually constitute small oligopeptides as signaling molecules (AIs) rather than the AHL which are predominant to gram negative bacteria produced through the cleavage of precursor proteins. The oligopeptides are cleaved into 10-20 functional signaling molecules composed of amino acids which are actively transported by specific protein transporters. As the signaling molecules attains the minimum concentration required to exert its signaling function, it diffuses out of the cell though the sensory protein spanning the cell surface [7]. Thus, the signaling molecule interacts with the protein sensor and undergoes phosphorylation with phosphate moiety binding to the regulatory protein. This binding result in the activation of the target genes at specific site of the DNA [13].

Even though the mechanism of quorum sensitivity in gram positive is different from that of gram negative bacteria, the technicalities concept remain the same. The number of AIs which are the peptides in the case of gram positive bacteria increases as the cell number increasing prior to the transmission of signals that encodes the activation of the Lux operon. and then the information is sent in to the DNA

\subsection{Quorum Sensing LuxS/AI-2 Circuit}

The third type of QS found in different bacterial species is synthesized by LuxS are responsible for inter Species communication between the bacterial species [14]. The synthesis begins with the use of S-adenosyl-L-homocysteine 
(SAH), which is a common product of SAM (S-adenosyl-Lmethionine) [15]. The S-adenosyl-L-homocysteine ( $\mathrm{SAH})$ which is found in large number of bacterial species is later hydrolysed to SRH (S-ribosyl-L-homocysteine) [15]. LuxS catalyses the cleavage of S-ribosyl-L-homocysteine (SRH) into homocysteine and 4, 5-dihydrody-2, 3-pentanedione (DPD) [16]. 4,5-Dihydrody-2,3-pentanedione interacts with water and cyclises into furanone compound due to the facts that unstable[4].
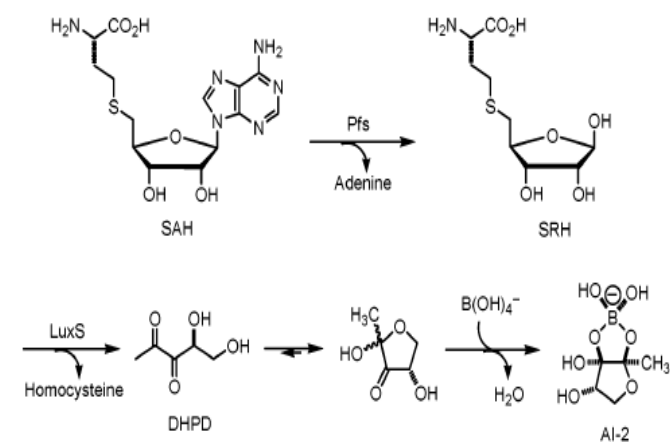

Figure 1. Biosynthesis of AI-2. The conversion of S-ribosylhomocysteine (SRH) to homocysteine and DPD is accompanied by LuxS enzyme. DPD is spontaneously converted into AI-2 molecule from [17].

This type of quorum sensing which involves the use of two AIs was first observed Vibrio harveyi, $a$ free luminous bacterium. The two types of AIs function parallel to each other [13] to control the expression of luciferase operon LuxCDABE which is always density dependent [18]. This complex QS system has some structures which are commonly found in Gram positive and gram negative bacteria [19] and it has been detected in a large number of bacteria species using the sequence analysis [4].

Vibrio harveyi also respond to AHL like the other gram negative counterpart as AI-1 and LuxS was found to be the AI-2; it is highly homologous and was detected in almost half of all bacterial sequence genome [20]. The AI-1 and AI-2 are recognised by signaling sensor protein, LuxN and LuxQ respectively. They usually have a transmembrane domain which connects them from the outside environment, thus responsible in sending information from the outside into the cell using a phosphorylation cascade that always involved the use of histidine and aspartic acid[18, 21]. In system 1, the periplasmic binding protein called LuxM, an AHL signal synthase interact with LuxN to response to AHL signal while in system 2, luxP interact with LuxQ to recognised AI2[22]. LuxN and LuxQ sensor acts as kinases when the concentration of the AI is low and this makes them to autophospholates their histidine conserved residues (H1) due to the absent of ligands and subsequently and transferred the phosphate to the next conserved aspartic residue (D1) each in their respective sensor domain.

The phosphate from both LuxN and LuxQ are then channeled to the next histidine conserved residue (H2) circuit to protein called LuxU, then to the aspartic residue (D2) of LuxO. Phosphorylation LuxO is active which result in the production of small regulatory RNA with Hfq chaperone destabilize LuxR and preventing the expression mRNA encoding it, thus switch off light production[23]. Transduction of signal to LuxO protein control luciferase expression in Vibrio harveyi [18].

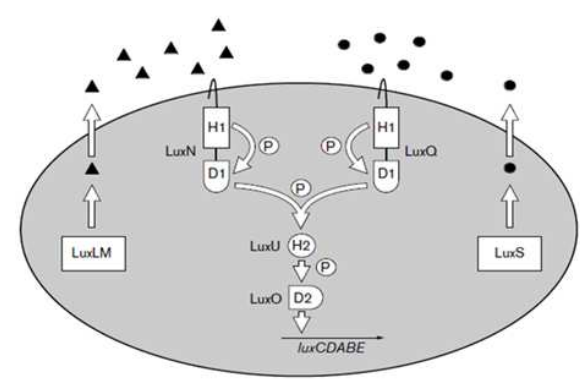

Figure 2. Quorum sensing in V. harveyi showing the two types of the AI, HAI1 synthesize by LuxLM and AI2 synthesize by LuxS. [1879).

In contrast, at $\mathrm{HCD}$, that is when the population of the AI1 and AI-2 are sufficiently high, the LuxN and LuxQ flip a switch and change from being kinases to phosphatise. The phosphate flow back through the circuit fom LuxO to LuxU to LuxQ and finally reaches LuxQ where it is finally hydrolysed. Thus, dephosphore LuxO is inactive and cannot activates the small regulatory RNA and this makes the LuxR mRNA to remain stable[23].

This stability of the LuxR mRNA result in the formation of LuxR, thus activating the transcription of luciferase operon and produces light [24]. LuxR is a homologue of HapR protease regulatory protein[25] in Vibrio cholerae which repressed the and toxin coregulated pilus (TCP) and Cholera toxin (CT) virulence gene [25].

\section{Quorum Sensing Control of Vibrio Cholerae Virulence}

Vibrio cholerae is a gram negative bacteria, a causative agent of cholera which is characterised by profuse acute dehydration as a result of severe watery diarrhoea which is endemic to many developing countries [26]. Rapid dehydration that is associated with cholera patient can result to a loss of up to 20 litres of body fluid within 24 hours and more than half of the percentage die without treatment[27]. The estimated annual worldwide burden associated with cholera infection reaches about several million cases [28]. It usually infects human being through the ingestion of contaminated food or where the supplies of clean water are compromised.

The main symptoms of this infection include severe water diarrhoea which can subsequently result to dehydration and eventually dead if not properly treated [2]. Other environment in which the Vibrio cholerae can be found include the coastal and estuarine areas which are normally found in proximity to dense human population [29]. It is a facultative pathogen which is found in a wide range of environments such as the human host where it survive harsh condition in the gastrointestinal tract (GIT) before finally 
colonizing the small intestine where it produces two enterotoxin chemicals which are responsible for the severe diarrhoea [30].

In Vibrios species, QS communication are usually controlled by three signaling molecules whose production in turn is also controlled by three of their key enzymes [31]. Cholera AI-1 (CAI-1), AHL and AI-2 signaling molecules generally controlled CqSA, LuxN and LuxS enzymes respectively. While in human Vibrio cholerae, CAI-1 and AI-2 signaling molecules are used to activate their QS[24]. Subsequent binding of the signaling molecules through their receptors result in the induction of Vibrio cholerae phosphorelay cascade, thus resulting in activation of certain genes including some virulence gene[23]. The major virulence factor for Vibrio cholerae are the Cholera toxin (CT) and Toxin coregulated pilus (TCP) and this are regulated as part of ToxR regulon [7].

This virulence signal transduction cascade play a vital role in detecting and integrating information from the environment and at the same time controlling the regulon for virulence [25]. One of the unique future significance of Vibrio Cholerae is that it uses QS to turn off virulence which is in contrast with other pathogenic bacteria which uses it to cause infection. The control of virulence in Vibrio Cholerae involves the use of three parallel QS circuit which are responsible for detecting the presence of $\mathrm{AI}$ and transmitting of information together to achieve a specific target [32]. In a study reported by Miller et al., (2002) virulence such as the CT and TCP and the formation of biofilm are rather repressed rather than activated at $\mathrm{HCD}$ which is reversed among the pathogenic bacteria.

A mouse model proposed by Hammer and Bassler, (2003) demonstrated that early colonization stage of infection (LCD), virulence CT and TCP genes are expressed which is due to the repression of HapR gene by the active LuxO. Active phospho LuxO indirectly represses HapR and activates the expression of AphA gene [25] and these allows for the colonization of the intestinal virulence genes together with biofilm formation. When the bacteria increase in size or multiply (HCD) at the later infection rate, the AIs which are the QS signals accumulate and activates the two cognate sensors system which in turn deactivates the LuxO. HapR is produced when LuxO is inactive which represses the expression of the virulence genes by the production of protease called HapA.

In general, HapR is a necessary requirement for the production of HA protease which encodes for the HapA virulence gene that serve as a detachase (scissors) which result in the removal of the bacteria from the intestinal tissue after colonization.

The signaling mechanism of Vibrio cholerae to some extent are similar to that of Vibrio harveyi counterpart which produce and response to 2 types of AIs connected using 2 parallel circuit[22]. The AI-1 of the Vibrio cholera is (S)-3-hyroxytridecan-4-one (CAI-1) synthesized by CqSA enzyme using SAM and decanoyl-coenzyme A that served as their substrate.

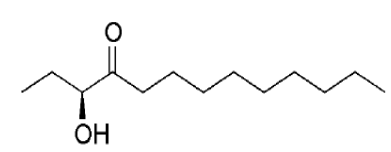

Figure 3. Vibrio cholerae AII (S)-3-hyroxytridecan-4-one (CAI-1)

The CqSA synthase and it sensor called CqsS has a homologue with Vibrio harveyi, which is LuxN and the AI for this system is called CAI-1[7]. It was reported by Miller et al., 2002 that CAI-1 plays a vital as an inter genus communication molecule due to the identification of homologue of CqSA among the vibrios species, thus allowing communication between them. CqsA is an aminotransferase enzyme that responsible for the production of CAI-1[33].

As mentioned earlier, Vibrio cholerae have 2 parallel QS, the AI-2 is synthesized by LuxS as found in other bacteria species. Vibrio cholerae just as Vibrio harveyi also has LuxS, a metabolic enzyme that play a vital role in the synthesis of AI-2. The enzymatic activity of the LuxS converts S-ribosylhomocysteine, an intermediate of SAM cycle to 4, 5-dihydroxy- 2, 3-penanedionine (DPD) and homocysteine. The DPD which is usually not a stable compound is later spontaneously converted into AI-2 [34]. Homologue of LuxS have been reported in many gram negative and positive bacteria and this facilitates interspecies communication among the bacteria population, thus enhancing the survival and cooperation among different species [35].

\section{Mehanism of Activation and Regulation in Vibrio Cholera}

Vibrio cholerae detects CAI-1 and AI-2 signals using two parallel membrane bound receptors which are transduced by sensor kinases CqsS and LuxP/LuxQ complex, respectively [36].

The CqsS and LuxQ receptors act as kinases in the absence of ligand, at low cell density, in the photophosphorylate themselves. Upon the phosphorylation, the sensors transfer phosphate to LuxO after channelling through LuxU. Phosphorylation of LuxO result in the activation of genes which encodes the four regulatory RNAs called Qrr1-4 that interacts with the sRNA binding protein Hfq and the RNA chaperone [36]. All the four RNAs are very essential in quorum sensing repression even though any one of them is sufficient enough for proper functioning of the system[37].

It was reported by Rutherford and bassler (2012) that up to date, the four regulatory RNA Qrr (qrr1-4) promoters and the luxO promoters are the only known target for the regulation of LuxO. LuxO can function as either an activator or repressor depending on the condition it found itself. When acting as an activator, the alternative sigma $54\left(\delta^{54}\right)$ is required and this is responsible for the transcription of the Qrr RNA gene and thus, not required when it is acting as repressors [37]. The main target of the small RNA Qrr are 
the mRNAs which encodes the master Quorum Sensing regulators which are the HapR and AphA [38]. HapR and AphA each plays a role in the controlling of transcription of hundreds of downstream genes[28].

At LCD, active phosphorylated LuxO activates the small regulaory RNA Qrr (qrr1-4)[39]. The small regulatory RNA together with $\mathrm{Hfq}$ Chaperone binds to HapR mRNA transcript and destabilise it, thus resulting in the activation of AphA mRNA transcript, a master regulon at LCD [38]. As a result, HapA protein is absent while AphA protein is made [28]. Expression of AphA activates tcpPH. Activated tcpPH activates toxT expression which further activates the major virulence factors [2]. Apart from the activation of the major virulence factors which are the CT and TCP genes which are express at low level of HapR, as well as vps genes involved in biofilm formation, without the exception of Hap proteses expression called HapA[7].

In Vibrio cholerae, AphA is the master regulator that operates at LCD while the HapR in contrast is the master regulator at $\mathrm{HCD}[38]$. The AphA which is a helix transcription factor with LysR type of another transcription called AphB work together to activates the transcription of tcpPH [2]. tcpP has a DNA membrane binding and periplasmic domain which requires the presence of tcpH, another binding protein that enhance it function by interacting through it periplasmic domain [27]. Together, tcpPH activates the expression of toxT, a transcription factor that plays a major role in the activation of the genes which specifically encodes the major Vibrio cholerae virulence factor, CT and TCP [2]. ToxT which is a member of AraC large protein has a binding domain at it $\mathrm{C}$ terminus [27] is also activated by ToxRS, a tcpPH homologue.

Expression of HapR also represses the expression of biofilm formation by a mechanism which is not well understood by binding to the aphA promoter and repressing it Transcription[40]. The base pair formed between the Qrr RNA and the HapR mRNA overlap the ribosome binding sequence (RBS) thereby preventing the ribosome to bind, thus facilitating the degradation of the HapR mRNA[2]. In general term, at LCD, the Qrr sRNAs repress the expression of hapR and at the same time activate the expression of AphA. The activation of AphA and the repression of HapR activates the expression of all the Vibrio Cholerae virulence genes as mentioned above [7].

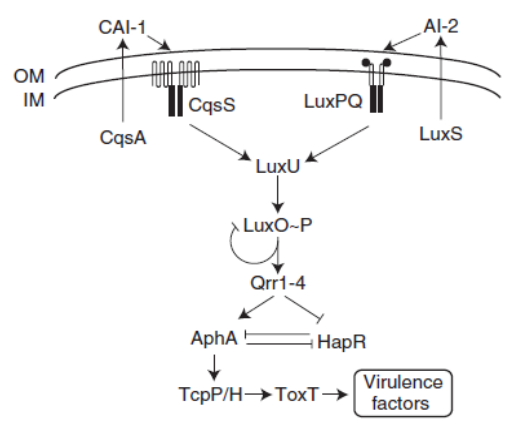

Figure 4. V. cholerae detects CAI-1 and AI-2 which are produced by CqsA and LuxS repectively via two two-component histidine kinases, CqsS and LuxPQ [2].
At high cell density, the accumulated CAI-1 and AI2 will bind to their CsqS and LuxPQ receptors respectively which reverses the phosphate flow by switching them from kinases to phosphatises which result in dephosphorylation or inactivation of LuxO [7]. The inactivated LuxO which is inactive cannot bind to the small RNA and this result to the expression of HapR. The expression of HapR represses the expression of the Vibrio Cholerae virulence genes such as the CT and TCP. Also, the at HCD, HapR expression play a vital role in the biofilm formation by directly repressing the gene that encodes the component of biofilm factory and also represses the two transcription factors which activates the genes VpsR and VpsT, responsible for the formation of the biofilm [2]. It was reported by Kovacikova and Skorupski (2002) that at HCD, the LuxO is inactive and cannot repressed the HapR gene and this will cause the HapR to influence the virulence cascade by binding to the recognition site of the AphA promoter and thereby repressing or decreasing the expression. Also in the work reported by Zhu et al., (2002) shows that the HapR play a vital role in controlling the expression of virulence factors in Vibrio cholera Rutherford et al., (2011) reported that HapR is the master regulator at high cell density which represses the AphA to shut down all virulence gene.

\section{Chemical inhibitors and agonist of Vibrio cholera}

The massive increase in Vibrio cholerae disease in humans more especially in developing counties has resulted in growing interest in finding an alternative way to control such infection. The present novel means is through the use of chemicals that serves as either inhibitors or antagonist which interfere with the bacterial communication [15]. These chemicals disrupt the normal bacterial communication by inhibiting the proper function of AI-2 by interfering with the DNA binding activities in all the Vibro species. Cinnamaldehyde and furanone derivatives are chemicals which inhibits the proper function of AI-2 in response to LuxR regulator by decreasing it DNA binding ability[23]. Other chemicals such as S-homoribosyl-L-cysteine and S-anhydroribosyl-L homocysteine inhibits the function of LuxS enzyme which inturn affects AI-2 by blocking it production [41].

In addition to other chemicals which inhibit the proper function AI-2, there are others which inhibit the proper function of LuxO, the central QS regulator that regulates the transcription of Qrr sRNA genes. The identification of the thio azauracil derivatives compound by $\mathrm{Ng}$ et al., 2012 play a vital role in the inhibition of ATP hydrolysis. Molecules from the family of this compound competitively bind to the performed LuxO-ATP complex and inhibiting ATP hydrolysis, thus preventing it transcription.

Apart from chemical inhibitors that are associated with Vibrio cholerae, there exist some chemicals which also function as agonist in the phosphorylation cascade. The CAI-1 which was found to be (S)-3-hydroxytridecan-4-one also have their agonist analogue. In a work reported by Nanting et al., 2009 shows that a synthesiszed chemical of R 
and $\mathrm{S}$ isomer functions effectively as a natural CAI-1, thus functioning as an analogue.

\section{Conclusion}

Manipulation of QS which involves the use of inhibitors or antagonist to block it has been recognized as a viable way for the development of novel therapeutics in the treatment of bacterial infection. Inhibitors are the compound which blocks the activities of enzyme while the compound which blocks the actions of the enzyme by binding to the receptors is the antagonist. As Bacterial use QS to cause various pathologically relevant events, it is believe that used of inhibitors could have vast therapeutic application. However the used of QS inhibitors and antagonist to tackle pathogenesis may help to turn off virulence and biofilm formation in many bacterial species.

In the use of QS to control virulence, several factors have to be taken into recognition depending on specific circumstances. Even though, the use of antagonist and inhibitors are commonly used in bacterial species which causes virulence at high cell density. Contrary to most of other bacteria species, Vibrio cholerae expresses virulence factors and form biofilm at low cell density i.e. at high cell density of CAI-1, virulence factor and biofilm formation are repressed.

The discovery of CAI-1 analogue could be used as in therapeutic applications such as in the repressing the production of virulence factor TCP. Therefore with this conceivable knowledge, it may be possible to design a drug with CAI-1 agonist analogue for the treatment of cholera infection.

Also, the identification of the synthetic ligand of CqsC receptor molecules could play a vital role in the determination of signal mechanism in Vibrio cholerae. The work reported by $\mathrm{Ng}$ et al., (2010) show that $\mathrm{CqsC}$ receptor does not detect or response to CAI-1 with shortened tail or bulky head groups. With such discovery, it implies such molecules are antagonist of $\mathrm{CqsC}$ receptors. With the fact that Vibrio cholerae causes virulence in the absence of AIs (i.e. at $\mathrm{LCD}$ ), $\mathrm{CqsC}$ receptors could serve as an interesting point in preventing virulence factor expression due to the fact that compound which triggers QS (i.e. potent $\mathrm{CqSC}$ receptor agonist) can be used to perform such function.

Finally, in addition to the use of CAI-1 analogue and receptors to control virulence, the use of thio azauracil derivatives compound can be used as prophylactic as they competitively bind with LuxO to inhibit ATP hydrolysis. Therefore, together with the use of receptors, CAI-1 analogue and any other QS activating molecules looks promising in combating of Vibrio cholerae infection and this could be of great relevant more especially in developing countries where it is endemic.

\section{References}

[1] Wang, L., J. Li, J.C. March, J.J. Valdes, and W.E. Bentley, luxS-dependent gene regulation in Escherichia coli $\mathrm{K}-12$ revealed by genomic expression profiling. Journal of bacteriology, 2005. 187(24): p. 8350-8360.

[2] Rutherford, S.T. and B.L. Bassler, Bacterial quorum sensing: its role in virulence and possibilities for its control. Cold Spring Harbor perspectives in medicine, 2012. 2(11).

[3] Bejerano-Sagie, M. and K.B. Xavier, The role of small RNAs in quorum sensing. Current opinion in microbiology, 2007. 10(2): p. 189-198.

[4] Antunes, L.C.M., R.B. Ferreira, M.M. Buckner, and B.B. Finlay, Quorum sensing in bacterial virulence. Microbiology, 2010. 156(8): p. 2271-2282.

[5] González, J.E. and N.D. Keshavan, Messing with bacterial quorum sensing. Microbiology and Molecular Biology Reviews, 2006. 70(4): p. 859-875.

[6] Mohammed, J., B.M. Abubakar, H. Yusuf, M. d Sulaiman, H. Saidu, A. Idris, and H. Tijani, Bacterial Biofilm: A Major Challenge of Catheterization. J. of Microbiology Res, 2013. 3(6): p. 213-223.

[7] Kaper, J.B. and V. Sperandio, Bacterial cell-to-cell signaling in the gastrointestinal tract. Infection and immunity, 2005. 73(6): p. 3197-3209.

[8] Li, Y.-H. and X. Tian, Quorum sensing and bacterial social interactions in biofilms. Sensors, 2012. 12(3): p. 2519-2538.

[9] Schaefer, A.L., D.L. Val, B.L. Hanzelka, J.E. Cronan Jr, and E. Greenberg, Generation of cell-to-cell signals in quorum sensing: acyl homoserine lactone synthase activity of a purified Vibrio fischeri LuxI protein. Proceedings of the National Academy of Sciences, 1996. 93(18): p. 9505-9509.

[10] Choi, J., D. Shin, M. Kim, J. Park, S. Lim, and S. Ryu, LsrR-mediated quorum sensing controls invasiveness of Salmonella typhimurium by regulating SPI-1 and flagella genes. PloS one, 2012. 7(5): p. e37059.

[11] Atkinson, S. and P. Williams, Quorum sensing and social networking in the microbial world. Journal of The Royal Society Interface, 2009. 6(40): p. 959-978.

[12] Popham, D.L. and A.M. Stevens, Bacterial Quorum Sensing and Bioluminescence. 2006.

[13] Miller, M.B. and B.L. Bassler, Quorum sensing in bacteria. Annual Reviews in Microbiology, 2001. 55(1): p. 165-199.

[14] Jacobi, C.A., S. Grundler, C.-J. Hsieh, J.S. Frick, P. Adam, G. Lamprecht, I.B. Autenrieth, M. Gregor, and P. Malfertheiner, Quorum sensing in the probiotic bacterium Escherichia coli Nissle 1917 (Mutaflor)-evidence that furanosyl borate diester (AI-2) is influencing the cytokine expression in the DSS colitis mouse model. Gut pathogens, 2012. 4(1): p. 8.

[15] Ni, N., M. Li, J. Wang, and B. Wang, Inhibitors and antagonists of bacterial quorum sensing. Medicinal research reviews, 2009. 29(1): p. 65-124. 
[16] Ruzheinikov, S., S. Das, S. Sedelnikova, A. Hartley, S. Foster, M. Horsburgh, A. Cox, C. McCleod, A. Mekhalfia, and G. Blackburn, The 1.2 A structure of a novel quorum-sensing protein, Bacillus subtilis LuxS. Journal of molecular biology, 2001. 313(1): p. 111-122.

[17] Zhu, J., X. Hu, E. Dizin, and D. Pei, Catalytic mechanism of S-ribosylhomocysteinase (LuxS): direct observation of ketone intermediates by $13 C$ NMR spectroscopy. Journal of the American Chemical Society, 2003. 125(44): p. 13379-13381.

[18] Bassler, B.L., How bacteria talk to each other: regulation of gene expression by quorum sensing. Current opinion in microbiology, 1999. 2(6): p. 582-587.

[19] Agarwal, R., S. Lata, M. Gupta, and P. Singh, Removal of melanoidin present in distillery effluent as a major colorant: a review. Journal of Environmental Biology, 2010. 31(4): p. 521-528.

[20] Khmel, I. and A. Metlitskaya, Quorum sensing regulation of gene expression: A promising target for drugs against bacterial pathogenicity. Molecular Biology, 2006. 40(2): p. 169-182.

[21] Tijani, H.I., I. Abdulrahman, B.M. Abubakar, S. Mohammed, J.N. Mohammed, H. Saidu, and H. Yusuf, Genetic proclivities of two-component modulated aerobiosis (2014). Vol. 2, No. 1, 2014, pp. 1-6

[22] Cámara, M., A. Hardman, P. Williams, and D. Milton, Quorum sensing in Vibrio cholerae. nature genetics, 2002. 32(2): p. 217-218.

[23] Brackman, G., T. Defoirdt, C. Miyamoto, P. Bossier, S. Van Calenbergh, H. Nelis, and T. Coenye, Cinnamaldehyde and cinnamaldehyde derivatives reduce virulence in Vibrio spp. by decreasing the DNA-binding activity of the quorum sensing response regulator LuxR. BMC microbiology, 2008. 8(1): p. 149.

[24] Miller, M.B., K. Skorupski, D.H. Lenz, R.K. Taylor, and B.L. Bassler, Parallel Quorum Sensing Systems Converge to Regulate Virulence in Vibrio cholerae. Cell, 2002. 110(3): p. 303-314.

[25] Zhu, J., M.B. Miller, R.E. Vance, M. Dziejman, B.L. Bassler, and J.J. Mekalanos, Quorum-sensing regulators control virulence gene expression in Vibrio cholerae. Proceedings of the National Academy of Sciences, 2002. 99(5): p. 3129-3134.

[26] Tsou, A.M., T. Cai, Z. Liu, J. Zhu, and R.V. Kulkarni, Regulatory targets of quorum sensing in Vibrio cholerae: evidence for two distinct HapR-binding motifs. Nucleic acids research, 2009. 37(8): p. 2747-2756.

[27] Matson, J.S., J.H. Withey, and V.J. DiRita, Regulatory networks controlling Vibrio cholerae virulence gene expression. Infection and immunity, 2007. 75(12): p. 5542-5549.

[28] Ng, W.-L., L. Perez, J. Cong, M.F. Semmelhack, and B.L. Bassler, Broad Spectrum Pro-Quorum-Sensing Molecules as Inhibitors of Virulence in Vibrios. PLoS pathogens, 2012. 8(6): p. e1002767.
[29] Joelsson, A., B. Kan, and J. Zhu, Quorum sensing enhances the stress response in Vibrio cholerae. Applied and environmental microbiology, 2007. 73(11): p. 3742-3746.

[30] Faruque, S.M., M.J. Albert, and J.J. Mekalanos, Epidemiology, Genetics, and Ecology of ToxigenicVibrio cholerae. Microbiology and molecular biology reviews, 1998. 62(4): p. 1301-1314.

[31] Brackman, G., S. Celen, U. Hillaert, S. Van Calenbergh, P. Cos, L. Maes, H.J. Nelis, and T. Coenye, Structure-activity relationship of cinnamaldehyde analogs as inhibitors of AI-2 based quorum sensing and their effect on virulence of Vibrio spp. PloS one, 2011. 6(1): p. e16084.

[32] Kovacikova, G. and K. Skorupski, Regulation of virulence gene expression in Vibrio cholerae by quorum sensing: HapR functions at the aphA promoter. Molecular microbiology, 2002. 46(4): p. 1135-1147.

[33] Ng, W.-L., Y. Wei, L.J. Perez, J. Cong, T. Long, M. Koch, M.F. Semmelhack, N.S. Wingreen, and B.L. Bassler, Probing bacterial transmembrane histidine kinase receptor-ligand interactions with natural and synthetic molecules. Proceedings of the National Academy of Sciences, 2010. 107(12): p. 5575-5580.

[34] Surette, M.G., M.B. Miller, and B.L. Bassler, Quorum sensing in Escherichia coli, Salmonella typhimurium, and Vibrio harveyi: a new family of genes responsible for autoinducer production. Proceedings of the National Academy of Sciences, 1999. 96(4): p. 1639-1644.

[35] Federle, M.J. and B.L. Bassler, Interspecies communication in bacteria. Journal of Clinical Investigation, 2003. 112(9): $\mathrm{p}$. 1291-1299.

[36] von Bodman, S.B., J.M. Willey, and S.P. Diggle, Cell-cell communication in bacteria: united we stand. Journal of bacteriology, 2008. 190(13): p. 4377-4391.

[37] Lenz, D.H., K.C. Mok, B.N. Lilley, R.V. Kulkarni, N.S. Wingreen, and B.L. Bassler, The Small RNA Chaperone Hfq and Multiple Small RNAs Control Quorum Sensing in Vibrio harveyi and Vibrio cholerae. Cell, 2004. 118(1): p. 69-82.

[38] Rutherford, S.T., J.C. van Kessel, Y. Shao, and B.L. Bassler, AphA and LuxR/HapR reciprocally control quorum sensing in vibrios. Genes \& development, 2011. 25(4): p. 397-408.

[39] Hunter, G., F.G. Vasquez, and J. Keener, A mathematical model and quantitative comparison of the small RNA circuit in the Vibrio harveyi and Vibrio cholerae quorum sensing systems. Physical biology, 2013. 10(4): p. 046007.

[40] Hammer, B.K. and B.L. Bassler, Quorum sensing controls biofilm formation in Vibrio cholerae. Molecular microbiology, 2003. 50(1): p. 101-104.

[41] Shen, G., R. Rajan, J. Zhu, C.E. Bell, and D. Pei, Design and synthesis of substrate and intermediate analogue inhibitors of S-ribosylhomocysteinase. Journal of medicinal chemistry, 2006. 49(10): p. 3003-3011. 